\title{
Elliptic Systems with Nonlinearities of Arbitrary Growth
}

\author{
Djairo G. de Figueiredo and Bernhard Ruf
}

\begin{abstract}
In this paper we study the existence of nontrivial solutions for the following system of coupled semilinear Poisson equations:

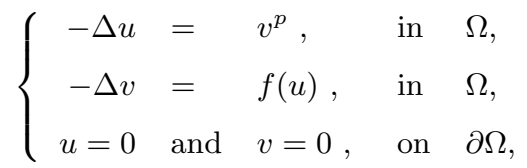

where $\Omega$ is a bounded domain in $\mathbb{R}^{N}$. We assume that $0<p<\frac{2}{N-2}$, and the function $f$ is superlinear and with no growth restriction (for example $f(s)=s e^{s}$ ); then the system has a nontrivial (strong) solution.
\end{abstract}

\section{Introduction}

We consider the system of equations

$$
\left\{\begin{array}{l}
-\Delta u=g(v), \text { in } \Omega \\
-\Delta v=f(u), \text { in } \Omega \\
\left.u\right|_{\partial \Omega}=\left.v\right|_{\partial \Omega}=0
\end{array},\right.
$$

where $\Omega$ is a bounded domain in $\mathbb{R}^{N}$. It is known, see [5], [11], [15], that for the "model case"

$$
f(s)=s^{q}, q>1, \text { and } g(s)=s^{p}, p>1,
$$

(here and in what follows, $s^{\alpha}:=\operatorname{sgn}(\mathrm{s})|s|^{\alpha}$ ) the system (1) has a nontrivial solution provided that

$$
1>\frac{1}{p+1}+\frac{1}{q+1}>1-\frac{2}{N}
$$

For $N=2$ this condition is satisfied for any $p>1$ and $q>1$.

For $N \geq 3$, the curve of $(p, q) \in \mathbb{R}^{2}$ satisfying $\frac{1}{p+1}+\frac{1}{q+1}=1-\frac{2}{N}$ is the so-called "critical hyperbola": for points $(p, q)$ on this curve one finds the typical problems of non-compactness, and non-existence of solutions, as it was proved in [23], [18], using Pohozaev type arguments.

\section{The case $\mathrm{N}=2$}

As mentioned above, for $N=2$ any pair of powers $(p, q) \in \mathbb{R}^{+} \times \mathbb{R}^{+}$satisfies the inequality (2). Actually, even a higher growth than polynomial is admitted: by the inequality of TrudingerMoser, see [22], [19], [20], subcritical growth for a single equation is given by the condition (see $[10])$

$$
\lim _{|t| \rightarrow \infty} \frac{g(t)}{e^{\alpha t^{2}}}=0, \forall \alpha>0
$$


It follows from a result in de Figueiredo-do Ó-Ruf [8] that system (1) has a non-trivial solution for nonlinearities $f$ and $g$ with such subcritical growth (and satisfying an AmbrosettiRabinowitz condition, see [2]). Also existence results for certain nonlinearities with critical growth are given in [8]. In this paper we consider a different type of extension of the known results: We will show that if one nonlinearity, say $g$, has polynomial growth (of any order), then, to prove existence of solutions, no growth restriction is required on the other nonlinearity $f$ (other than the Ambrosetti-Rabinowitz condition).

\section{The case $\mathrm{N}=3$}

Note that for $N=3$ the critical hyperbola has the asymptotes $p_{\infty}=2$ and $q_{\infty}=2$. In particular, if $g(s)=s^{p}$ with $1<p<2$, then the cited existence results say that there exists a solution $(u, v)$ for system (1) with $f(s)=s^{q}$, for any $q>1$. Also in this case we show that existence of solutions can be proved requiring no growth restriction whatsoever on the nonlinearity $f$ (other than the Ambrosetti-Rabinowitz condition).

\section{The case $\mathrm{N} \geq 4$}

For $N \geq 4$ the asymptotes of the critical hyperbola are in the values $p_{\infty}=\frac{2}{N-2} \leq 1$ and $q_{\infty}=\frac{2}{N-2} \leq 1$. Note that for an exponent $p<1$, the corresponding equation in the system is sublinear. i.e. we have a system with one sublinear and one superlinear equation. In this situation, the proposed approach is no longer applicable. However, in this case a reduction of the system to a single equation is possible (see Clément-Felmer-Mitidieri [6] and Felmer - Martínez [12]), which allows to prove again a result of the same form; moreover this approach also allows to extend to the whole range the cases $N=2$ and $N=3$, that is for $N=2: 0<p<+\infty$, and for $N=3: 0<p<2$.

The main result of the paper is stated in the following theorem:

Theorem 1.1. Suppose that

1) $g(s)=s^{p}$, with $\begin{cases}0<p, & \text { if } N=2 \\ 0<p<\frac{2}{N-2}, & \text { if } N \geq 3\end{cases}$

2) $f \in C(\mathbb{R})$, and set $F(s)=\int_{0}^{s} f(t) d t$;

- there exist constants $\theta>\left\{\begin{array}{l}2, \text { if } p \geq 1 \\ 1+\frac{1}{p}, \text { if } p<1\end{array}\right.$ and $s_{0} \geq 0$ such that $\theta F(s) \leq f(s) s, \forall|s| \geq s_{0}$

- and for $s$ near $0: \quad f(s)= \begin{cases}o(s), & \text { if } p \geq 1 \\ o\left(s^{1 / p}\right), & \text { if } p<1\end{cases}$

Then the system

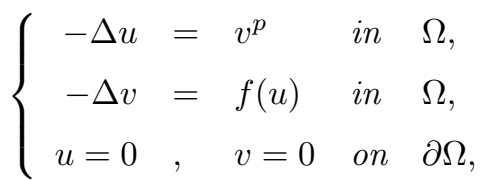

has a nontrivial (strong) solution.

\section{Remarks}

1) It is somewhat surprising that no growth restriction needs to be imposed on $f$, since for the single equation $-\Delta u=f(u)$ growth restrictions are, in general, necessary to prove the existence of solutions; we refer to the non-existence result in [9] for $N=2$, and to [20] for $N \geq 3$.

2) In the cases with $p>1$, the nonlinearity $g(s)=s^{p}$ may be replaced by more general functions, satisfying an Ambrosetti-Prodi type condition like $f(s)$, and the growth restriction

$$
|g(s)| \leq c|s|^{p}+d, \text { for some constants } c, d>0, \quad \text { and } \begin{cases}1<p, & N=2 \\ 1<p<2, & N=3\end{cases}
$$


For the sake of simplicity, we restrict here to the case $g(s)=s^{p}$.

For completeness we also state the following theorem:

Theorem 1.2. Suppose that

1) $(p, q)$ satisfy $\frac{1}{p+1}+\frac{1}{q+1}>1-\frac{2}{N}$, and $\frac{2}{N-2} \leq p \leq 1$.

2) $f \in C(\mathbb{R})$, and there exist constants $\theta>\frac{p+1}{p}$ and $s_{0} \geq 0$ such that

$$
\theta F(s):=\theta \int_{0}^{s} f(t) d t \leq f(s) s, \quad \forall|s| \geq s_{0}
$$

and

Then the system

$$
|f(s)| \leq c|s|^{q}+d, \text { for some constants } c, d>0
$$

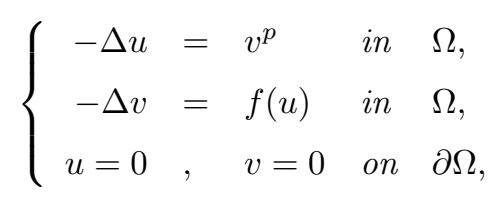

has a nontrivial (strong) solution.

In the literature we have only found the cases of $(p, q)$ below the critical hyperbola, and with the restriction that $p>1$ and $q>1$ (see [5], [15], [11]) and the case $0<p \cdot q<1$ (see Felmer-Martínez [12]). This does not cover the whole region below the critical hyperbola. The above theorem covers also the remaining cases below the critical hyperbola, namely

$$
0<p \leq 1 \text { and } p \cdot q \geq 1 \text {; }
$$

note that we need to make the restriction that the sublinear function $v^{p}$ is in the form of a power, while the superlinear function $f(u)$ may be of more general form.

\section{Proof: the case $p>1$}

In this section we consider the case $1<p<\frac{2}{N-2}$, i.e. $N=2,3$.

\subsection{The setting}

A natural functional associated to system (1) is

$$
J(u, v)=\int_{\Omega} \nabla u \nabla v d x-\int_{\Omega}(F(u)+G(v)) d x,
$$

with $F(s)=\int_{0}^{s} f(t) d t$ and $G(s)=\int_{0}^{s} g(t) d t$. The natural space to consider this functional is the Sobolev space $H_{0}^{1}(\Omega) \times H_{0}^{1}(\Omega)$; however, in order to have a well-defined $C^{1}$-functional on this space, one has to impose certain growth restrictions:

in $N=2: F$ and $G$ subcritical in the sense of Trudinger-Moser (see above)

in $N=3:|F(s)| \leq c|s|^{6}+d, \quad|G(s)| \leq c|s|^{6}+d$

These conditions are on the one hand too loose for $G(s)=\frac{1}{p+1} s^{p+1}$, where a more restrictive growth is given, and too strong on $F(s)$, where we do not want any growth limitation.

We therefore follow an idea of de Figueiredo-Felmer [11] and Hulshoff-vanderVorst [15], defining a related functional on suitable fractional Sobolev spaces.

Consider the Laplacian as the operator

$$
-\Delta: H^{2}(\Omega) \cap H_{0}^{1}(\Omega) \subset L^{2}(\Omega) \rightarrow L^{2}(\Omega),
$$

and $\left\{e_{i}\right\}_{i=1}^{\infty}$ a corresponding system of orthogonal and $L^{2}$-normalized eigenfunctions, with eigenvalues $\left\{\lambda_{i}\right\}$. Then, writing

$$
u=\sum_{n=1}^{\infty} a_{n} e_{n}, \text { with } a_{n}=\int_{\Omega} u e_{n} d x,
$$


we set

$$
E^{s}=\left\{u \in L^{2}(\Omega): \sum_{n=1}^{\infty} \lambda_{n}^{s}\left|a_{n}\right|^{2}<\infty\right\}
$$

and define a linear operator on $L^{2}(\Omega)$ by

$$
A^{s} u=\sum_{n=1}^{\infty} \lambda_{n}^{s / 2} a_{n} e_{n}, \forall u \in D\left(A^{s}\right):=E^{s} .
$$

The spaces $E^{s}$ are fractional Sobolev spaces with the inner product

$$
(u, v)_{s}=\int_{\Omega} A^{s} u A^{s} v d x,
$$

see Lions-Magenes [16], and we have

$$
\begin{aligned}
& E^{s}=H^{s}(\Omega) \text { if } 0 \leq s<\frac{1}{2}, \quad E^{1 / 2} \subset H^{1 / 2}(\Omega), \\
& E^{s}=\left\{u \in H^{s}(\Omega)|u|_{\partial \Omega}=0\right\} \quad \text { if } \quad \frac{1}{2}<s \leq 2, s \neq \frac{3}{2}, \text { and } \\
& E^{3 / 2} \subset\left\{u \in H^{3 / 2}(\Omega)|u|_{\partial \Omega}=0\right\}
\end{aligned}
$$

By the Sobolev imbedding theorem we therefore have continuous imbeddings

$$
E^{s} \subset L^{p}(\Omega), \text { if } \frac{1}{p} \geq \frac{1}{2}-\frac{s}{N},
$$

and these imebbedings are compact if $\frac{1}{p}>\frac{1}{2}-\frac{s}{N}$.

\subsection{The functional}

With these definitions, we now define the Hilbert space $E:=E^{t} \times E^{s}$, endowed with the norm

$$
\|(u, v)\|_{E}=\left(\|u\|_{E^{t}}^{2}+\|v\|_{E^{s}}^{2}\right)^{\frac{1}{2}}
$$

On the space $E$ we consider the functional

$$
\begin{aligned}
& I: E \rightarrow \mathbb{R}, \\
& I(u, v)=\int_{\Omega} A^{t} u A^{s} v-\int_{\Omega}\left(\frac{1}{p+1}|v|^{p+1}+F(u)\right) d x
\end{aligned}
$$

with $s$ and $t$ such that $s+t=2$; loosely speaking, this means that we distribute the two derivatives given in the first term of the functional $J$, see (5), differently on the variables $u$ and $v$. Of course, it is crucial to recuperate from critical points $(u, v)$ of this functional solutions of system (3). We state this in the following

Proposition 2.1. Suppose that $(u, v) \in E^{t} \times E^{s}$ is a critical point of the functional I, i.e. $u$ and $v$ are weak solutions of the system

$$
\left\{\begin{array}{l}
\int_{\Omega} A^{t} u A^{s} \phi=\int_{\Omega} v^{p} \phi, \forall \phi \in E^{s} \\
\int_{\Omega} A^{t} \psi A^{s} v=\int_{\Omega} f(u) \psi, \forall \psi \in E^{t} .
\end{array}\right.
$$

Then $v \in W^{2, \frac{p+1}{p}}(\Omega) \cap W_{0}^{1, \frac{p+1}{p}}(\Omega)$ and $u \in W^{2, q}(\Omega) \cap W_{0}^{1, q}(\Omega), \forall q \geq 1$, and hence $u$ and $v$ are "strong" solutions of (3), i.e.

$$
\left\{\begin{array}{l}
\int_{\Omega}(-\Delta u) \phi=\int_{\Omega} v^{p} \phi, \forall \phi \in C_{0}^{\infty}(\Omega) \\
\int_{\Omega}(-\Delta v) \psi=\int_{\Omega} f(u) \psi, \forall \psi \in C_{0}^{\infty}(\Omega) .
\end{array}\right.
$$


From this proposition follows by standard bootstrap arguments that $u$ and $v$ are classical solutions of (3) if $f$ and $\Omega$ are smooth.

The proof of this proposition follows ideas of de Figueiredo - Felmer [11], and will be given in subsection 2.5.

In the following subsection we prove that there exist values $s$ and $t$ with $s+t=2$ such that the functional $I$ is a well-defined $C^{1}$ functional, and that it has a non-trivial critical level.

\subsection{The choice of the spaces $E^{s}$ and $E^{t}$}

We begin by proving the following Lemma:

\section{Lemma 2.2.}

Let $1<p(N=2)$, or $1<p<2(N=3)$. Then there exist parameters $s>0$ and $t>0$ with $s+t=2$ such that the following embeddings are continuous and compact:

$$
E^{s}(\Omega) \subset L^{p+1}(\Omega) \quad, \quad E^{t}(\Omega) \subset C^{0}(\Omega)
$$

Proof. Note that $H^{s}(\Omega) \subset L^{p+1}(\Omega)$ compactly, iff $\frac{1}{p+1}>\frac{1}{2}-\frac{s}{N}$.

For $N=2$, we get thus the condition

$$
s>1-\frac{2}{p+1}
$$

Choose $s<1$ satisfying the previous condition, and set $t=2-s>1$. We have a compact embedding $E^{t}(\Omega) \subset C^{0}(\Omega)$ for

$$
\frac{t}{N}>\frac{1}{2}, \quad \text { i.e. for } t>1
$$

and hence the Lemma holds for $N=2$.

For $N=3$, we get the condition

$$
s>\frac{3}{2}-\frac{3}{p+1}
$$

Since

$$
\sup \left\{\frac{3}{2}-\frac{3}{p+1} \mid 1<p<2\right\}=\frac{1}{2},
$$

we can choose $s<\frac{1}{2}$, and then $t>\frac{3}{2}$, and hence $E^{t}(\Omega) \subset C^{0}(\Omega)$ compactly.

Thus, we now fix $s$ and $t$ as in Lemma 2.2, and define the functional $I(u, v)$ given by (6) on the space $E^{t} \times E^{s}=: E$.

In the next Lemma we collect a few properties of the operators $A^{s}$ and the spaces $E^{s}$.

Lemma 2.3. Let $s>0$ and $t>0$.

1) $z \in E^{s}$ iff $A^{s} z \in L^{2}$, and $\|z\|_{E^{s}}=\left\|A^{s} z\right\|_{L^{2}}$

2) Let $z \in E^{s+t}=E^{2}=H^{2}$; then $A^{s+t} z=A^{s} A^{t} z=A^{t} A^{s} z$.

Proof. 1) follows immediately from the definitions.

2) we have

$$
A^{s+t} z=\sum_{i \in \mathbb{N}} \alpha_{i} \lambda_{i}^{(s+t) / 2} e_{i}=\sum_{i \in \mathbb{N}} \alpha_{i} \lambda_{i}^{s / 2} \lambda_{i}^{t / 2} e_{i}=A^{s} \sum_{i \in \mathbb{N}} \alpha_{i} \lambda_{i}^{t / 2} e_{i}=A^{s} A^{t} z
$$




\subsection{Existence of a non-trivial critical point}

The functional $I(u, v): E=E^{t} \times E^{s}$ is strongly indefinite near zero, in the sense that there exist infinite dimensional subspaces $E^{+}$and $E^{-}$with $E^{+} \oplus E^{-}=E$ such that the functional is (near zero) positive definite on $E^{+}$and negative definite on $E^{-}$. Li-Willem [17] prove the following general existence theorem for such situations, which can be applied in our case:

Theorem 2.4 (Li-Willem, 1995).

Let $\Phi: E \rightarrow \mathbb{R}$ be a strongly indefinite $C^{1}$-functional satisfying

A1) $\Phi$ has a local linking at the origin, i.e. for some $r>0$ :

$$
\Phi(z) \geq 0 \text { for } z \in E^{+},\|z\|_{E} \leq r \quad, \quad \Phi(z) \leq 0, \text { for } z \in E^{-},\|z\|_{E} \leq r .
$$

A2) $\Phi$ maps bounded sets into bounded sets.

A3) Let $E_{n}^{+}$be any $n$-dimensional subspace of $E^{+}$; then $\phi(z) \rightarrow-\infty$ as $\|z\| \rightarrow \infty$, $z \in E_{n}^{+} \oplus E^{-}$.

A4) $\Phi$ satisfies the Palais-Smale condition (PS) (Li-Willem [17] require a weaker "(PS $)$ condition", however, in our case the classical (PS) condition will be satisfied).

Then $\Phi$ has a nontrivial critical point.

We now verify that our functional satisfies the assumptions of this theorem.

First, it is clear, with the choices of $s$ and $t$ made above, that $I(u, v)$ is a $C^{1}$-functional on $E^{s} \times E^{t}$.

A1) Following de Figueiredo-Felmer [11] we can define the spaces

$$
E^{+}=\left\{\left(u, A^{t-s} u\right) \mid u \in E^{t}\right\}, \text { and } E^{-}=\left\{\left(u,-A^{t-s} u\right) \mid u \in E^{t}\right\}
$$

which give a natural splitting $E^{+} \oplus E^{-}=E$. It is easy to see that $I(u, v)$ has a local linking with respect to $E^{+}$and $E^{-}$at the origin.

A2) Let $B \subset E^{t} \times E^{s}$ be a bounded set, i.e. $\|u\|_{E^{t}} \leq c,\|v\|_{E^{s}} \leq c$, for all $(u, v) \in B$. Then

$$
\begin{aligned}
|I(u, v)| & \leq\left\|A^{t} u\right\|_{L^{2}}\left\|A^{s} v\right\|_{L^{2}}+\int_{\Omega}|v|^{p+1}+\int_{\Omega}|f(u)| \\
& \leq\|u\|_{E^{t}}\|v\|_{E^{s}}+c\|v\|_{E^{s}}^{p+1}+\sup _{x \in \Omega}|f(u(x))| \cdot|\Omega| \leq C
\end{aligned}
$$

A3) Let $z_{k}=z_{k}^{+}+z_{k}^{-} \in E=E_{n}^{+} \oplus E^{-}$denote a sequence with $\left\|z_{k}\right\|_{E} \rightarrow \infty$. By the above, $z_{k}$ may be written as

$$
z_{k}=\left(u_{k}, A^{t-s} u_{k}\right)+\left(w_{k},-A^{t-s} w_{k}\right) \text {, with } u_{k} \in E_{n}^{t}, w_{k} \in E^{t},
$$

where $E_{n}^{t}$ denotes an $n$-dimensional subspace of $E^{t}$. Thus, the functional $I\left(z_{k}\right)$ takes the form

$$
\begin{aligned}
I\left(z_{k}\right)= & \int_{\Omega} A^{t} u_{k} A^{s} A^{t-s} u_{k}-\int_{\Omega} A^{t} w_{k} A^{s} A^{t-s} w_{k}- \\
& \quad-\frac{1}{p+1} \int_{\Omega}\left|A^{t-s}\left(u_{k}-w_{k}\right)\right|^{p+1}-\int_{\Omega} F\left(u_{k}+w_{k}\right) \\
= & \int_{\Omega}\left|A^{t} u_{k}\right|^{2}-\int_{\Omega}\left|A^{t} w_{k}\right|^{2}-\frac{1}{p+1} \int_{\Omega}\left|A^{t-s}\left(u_{k}-w_{k}\right)\right|^{p+1}-\int_{\Omega} F\left(u_{k}+w_{k}\right)
\end{aligned}
$$

Note that $\left\|z_{k}\right\| \rightarrow \infty \Longleftrightarrow \int\left|A^{t} u_{k}\right|^{2}+\int\left|A^{t} w_{k}\right|^{2}=\left\|u_{k}\right\|_{E^{t}}^{2}+\left\|w_{k}\right\|_{E^{t}}^{2} \rightarrow \infty$.

Now, if

1) $\left\|u_{k}\right\|_{E^{t}} \leq c$, then $\left\|w_{k}\right\|_{E^{t}} \rightarrow \infty$, and then $I\left(z_{k}\right) \rightarrow-\infty$

2) $\left\|u_{k}\right\|_{E^{t}} \rightarrow \infty$, then we estimate (c, $c_{1}$ and $c_{2}$ are positive constants) using the fact that $t-s>0$ and $p>1$

$$
\int_{\Omega}\left|A^{t-s}\left(u_{k}-w_{k}\right)\right|^{p+1} \geq c\left(\int_{\Omega}\left|A^{t-s}\left(u_{k}-w_{k}\right)\right|^{2}\right)^{\frac{p+1}{2}} \geq c_{1}\left\|u_{k}-w_{k}\right\|_{L^{2}}^{p+1}
$$


and

and hence we obtain the estimate

$$
\int_{\Omega} F\left(u_{k}+w_{k}\right) \geq c_{2} \int_{\Omega}\left|u_{k}+w_{k}\right|^{p+1}-d \geq c_{1}\left\|u_{k}+w_{k}\right\|_{L^{2}}^{p+1}-d
$$

$$
I\left(z_{k}\right) \leq \frac{1}{2}\left\|u_{k}\right\|_{E^{t}}^{2}-c_{1}\left(\left\|u_{k}-w_{k}\right\|_{L^{2}}^{p+1}+\left\|u_{k}+w_{k}\right\|_{L^{2}}^{p+1}\right)+d
$$

Since $\phi(t)=t^{p+1}$ is convex, we have $\frac{1}{2}(\phi(t)+\phi(s)) \geq \phi\left(\frac{1}{2}(s+t)\right)$, and hence

$$
\begin{aligned}
I\left(z_{k}\right) & \leq \frac{1}{2}\left\|u_{k}\right\|_{E^{t}}^{2}-c_{1} \frac{1}{2^{p}}\left(\left\|u_{k}-w_{k}\right\|_{L^{2}}+\left\|u_{k}+w_{k}\right\|_{L^{2}}\right)^{p+1}+d \\
& \leq \frac{1}{2}\left\|u_{k}\right\|_{E^{t}}^{2}-c_{1} \frac{1}{2^{p}}\left\|u_{k}\right\|_{L^{2}}^{p+1}+d
\end{aligned}
$$

Since on $E_{n}^{t}$ the norms $\left\|u_{k}\right\|_{E^{t}}$ and $\left\|u_{k}\right\|_{L^{2}}$ are equivalent, we conclude that also in this case $J\left(z_{k}\right) \rightarrow-\infty$.

A4) Let $\left\{z_{n}\right\} \subset E$ denote a (PS)-sequence, i.e. such that

$$
\left|I\left(z_{n}\right)\right| \rightarrow c, \quad \text { and }\left|\left(\Phi^{\prime}\left(z_{n}\right), \eta\right)\right| \leq \epsilon_{n}\|\eta\|_{E}, \forall \eta \in E, \quad \text { and } \epsilon_{n} \rightarrow 0
$$

We first show:

Lemma 2.5. The (PS)-sequence $\left\{z_{n}\right\}$ is bounded in $E$.

Proof. By (9) we have for $z_{n}=\left(u_{n}, v_{n}\right)$

$$
\begin{gathered}
I\left(u_{n}, v_{n}\right)=\int_{\Omega} A^{t} u_{n} A^{s} v_{n}-\frac{1}{p+1} \int_{\Omega} v_{n}^{p+1}-\int_{\Omega} F\left(u_{n}\right) \rightarrow c \\
I^{\prime}\left(u_{n}, v_{n}\right)(\phi, \psi)=\int_{\Omega} A^{t} u_{n} A^{s} \psi+\int_{\Omega} A^{s} v_{n} A^{t} \phi-\int_{\Omega} v_{n}^{p} \psi-\int_{\Omega} f\left(u_{n}\right) \phi=\epsilon_{n}\|(\phi, \psi)\|_{E}
\end{gathered}
$$

Choosing $(\phi, \psi)=\left(u_{n}, v_{n}\right) \in E^{t} \times E^{s}$ we get by $(11)$

$$
2 \int_{\Omega} A^{t} u_{n} A^{s} v_{n}-\int v_{n}^{p+1}-\int_{\Omega} f\left(u_{n}\right) u_{n}=\epsilon_{n}\left(\left\|u_{n}\right\|_{E^{t}}+\left\|v_{n}\right\|_{E^{s}}\right)
$$

and subtracting this from $2 I\left(u_{n}, v_{n}\right)$ we obtain, using assumption 2) of Theorem 1.1

$$
\left(1-\frac{2}{p+1}\right) \int_{\Omega} v_{n}^{p+1}+\left(1-\frac{2}{\theta}\right) \int_{\Omega} f\left(u_{n}\right) u_{n} \leq C+\epsilon_{n}\left(\left\|u_{n}\right\|_{E^{t}}+\left\|v_{n}\right\|_{E^{s}}\right)
$$

and thus

$$
\begin{gathered}
\int_{\Omega} v_{n}^{p+1} \leq C+\epsilon_{n}\left(\left\|u_{n}\right\|_{E^{t}}+\left\|v_{n}\right\|_{E^{s}}\right) \\
\int_{\Omega} f\left(u_{n}\right) u_{n} \leq C+\epsilon_{n}\left(\left\|u_{n}\right\|_{E^{t}}+\left\|v_{n}\right\|_{E^{s}}\right)
\end{gathered}
$$

Choosing $(\phi, \psi)=\left(0, A^{t-s} u_{n}\right) \in E^{t} \times E^{s}$ in (11) we get

and hence by Hölder

$$
\int_{\Omega}\left|A^{t} u_{n}\right|^{2}=\int_{\Omega} v_{n}^{p} A^{t-s} u_{n}+\epsilon_{n}\left\|A^{t-s} u_{n}\right\|_{E^{s}}
$$

$$
\left\|u_{n}\right\|_{E^{t}}^{2}=\left\|A^{t} u_{n}\right\|_{L^{2}}^{2} \leq\left(\int_{\Omega}\left|v_{n}\right|^{p+1}\right)^{\frac{p}{p+1}}\left(\int_{\Omega}\left|A^{t-s} u_{n}\right|^{p+1}\right)^{\frac{1}{p+1}}+\epsilon_{n}\left\|u_{n}\right\|_{E^{t}}
$$

Noting that

$$
\left(\int_{\Omega}\left|A^{t-s} u_{n}\right|^{p+1}\right)^{\frac{1}{p+1}} \leq c\left\|A^{t-s} u_{n}\right\|_{E^{s}}=c\left\|A^{t} u_{n}\right\|_{L^{2}}=c\left\|u_{n}\right\|_{E^{t}}
$$

we obtain, using (14)

$$
\left\|u_{n}\right\|_{E^{t}}^{2} \leq\left[C+\epsilon_{n}\left(\left\|u_{n}\right\|_{E^{t}}+\left\|v_{n}\right\|_{E^{s}}\right)\right]^{p /(p+1)} \cdot c\left\|u_{n}\right\|_{E^{t}}+\epsilon_{n}\left\|u_{n}\right\|_{E^{t}}
$$

and thus

$$
\left\|u_{n}\right\|_{E^{t}} \leq C+\epsilon_{n}\left(\left\|u_{n}\right\|_{E^{t}}+\left\|v_{n}\right\|_{E^{s}}\right)^{p /(p+1)}
$$


Similarly as above we note that $A^{s-t} v_{n} \in E^{t}$, and thus, choosing $(\phi, \psi)=\left(A^{s-t} v_{n}, 0\right) \in$ $E^{t} \times E^{s}$ in (11) we get

$$
\int_{\Omega}\left|A^{s} v_{n}\right|^{2}=\int_{\Omega} f\left(u_{n}\right) A^{s-t} v_{n}+\epsilon_{n}\left\|A^{s-t} v_{n}\right\|_{E^{t}} \leq\left\|A^{s-t} v_{n}\right\|_{\infty} \int_{\Omega}\left|f\left(u_{n}\right)\right|+\epsilon_{n}\left\|v_{n}\right\|_{E^{s}}
$$

Using that $\left\|A^{s-t} v_{n}\right\|_{E^{t}}=\left\|A^{s} v_{n}\right\|_{L^{2}}=\left\|v_{n}\right\|_{E^{s}}$, and the fact that $E^{t} \subset C^{0}$ we then obtain, using (15)

$$
\begin{aligned}
\left\|v_{n}\right\|_{E^{s}} & \leq c \int_{\Omega}\left|f\left(u_{n}\right)\right|+\epsilon_{n}=\int_{\left[\left|u_{n}\right| \leq s_{0}\right]} \max _{|t| \leq s_{0}}|f(t)|+\int_{\left[\left|u_{n}\right|>s_{0}\right]} f\left(u_{n}\right) u_{n}+\epsilon_{n} \\
& \leq C+\epsilon_{n}\left(\left\|u_{n}\right\|_{E^{t}}+\left\|v_{n}\right\|_{E^{s}}\right)
\end{aligned}
$$

Joining (16) and (17) we finally get

$$
\left\|u_{n}\right\|_{E^{t}}+\left\|v_{n}\right\|_{E^{s}} \leq C+2 \epsilon_{n}\left(\left\|u_{n}\right\|_{E^{t}}+\left\|v_{n}\right\|_{E^{s}}\right)
$$

Thus, $\left\|u_{n}\right\|_{E^{t}}+\left\|v_{n}\right\|_{E^{s}}$ is bounded.

With this it is now possible to complete the proof of the (PS)-condition: since $\left\|u_{n}\right\|_{E^{t}}$ is bounded, we find a weakly convergent subsequence $u_{n} \rightarrow u$ in $E^{t}$. Since the mappings $A^{t}: E^{t} \rightarrow L^{2}$ and $A^{-s}: L^{2} \rightarrow E^{s}$ are continuous isomorphisms, we get $A^{t}\left(u_{n}-u\right) \rightarrow 0$ in $L^{2}$ and $A^{t-s}\left(u_{n}-u\right) \rightarrow 0$ in $E^{s}$. Since $E^{s} \subset L^{p+1}$ compactly, we conclude that $A^{t-s}\left(u_{n}-u\right) \rightarrow 0$ strongly in $L^{p+1}$.

Similarly, we find a subsequence of $\left\{v_{n}\right\}$ which is weakly convergent in $E^{s}$ and such that $v_{n}^{p}$ is strongly convergent in $L^{\frac{p+1}{p}}$

Choosing $(\phi, \psi)=\left(0, A^{t-s}\left(u_{n}-u\right) \in E^{t} \times E^{s}\right.$ in (11) we thus conclude

$$
\int_{\Omega} A^{t} u_{n} A^{t}\left(u_{n}-u\right)=\int_{\Omega} v_{n}^{p} A^{t-s}\left(u_{n}-u\right)+\epsilon_{n}\left\|A^{t-s}\left(u_{n}-u\right)\right\|_{E^{s}}
$$

By the above considerations, the righthand-side converges to 0 , and thus

$$
\int_{\Omega}\left|A^{t} u_{n}\right|^{2} \rightarrow \int_{\Omega}\left|A^{t} u\right|^{2}
$$

Thus, $u_{n} \rightarrow u$ strongly in $E^{t}$.

To obtain the strong convergence of $\left\{v_{n}\right\}$ in $E^{s}$, one proceeds similarly: as above, one finds a subsequence $\left\{v_{n}\right\}$ converging weakly in $E^{s}$ to $v$, and then $A^{s-t} v_{n} \rightarrow A^{s-t} v$ weakly in $A^{t}$ and $A^{s-t} v_{n} \rightarrow A^{s-t} v$ strongly in $C^{0}$. Choosing in $(9)(\phi, \psi)=\left(A^{s-t}\left(v_{n}-v\right), 0\right)$, we get

$$
\int_{\Omega} A^{s}\left(v_{n}-v\right) A^{s} v_{n}=\int f\left(u_{n}\right) A^{s-t}\left(v_{n}-v\right)+\epsilon_{n}\left(\left\|A^{s-t}\left(v_{n}-v\right)\right\|\right)
$$

The first term on the right is estimated by $\left\|A^{s-t}\left(v_{n}-v\right)\right\|_{C^{0}} \int_{\Omega}\left|f\left(u_{n}\right)\right| \rightarrow 0$, and thus one concludes again that

$$
\int_{\Omega}\left|A^{s} v_{n}\right|^{2} \rightarrow \int_{\Omega}\left|A^{s} v\right|^{2}
$$

and hence also $v_{n} \rightarrow v$ strongly in $E^{s}$.

Thus, the conditions of Theorem 2.4 are satisfied; hence, we find a positive critical point $(u, v)$ for the functional $I$, which yields a weak solution to system (3). 


\subsection{Strong solutions}

In this section we prove Proposition 2.1.

Consider the first equation in the system (7). We can follow the arguments of [11]: If $\phi \in H^{2}(\Omega) \cap H_{0}^{1}(\Omega)$, then

$$
\int_{\Omega} A^{t} u A^{s} \phi=\int_{\Omega} u A^{2} \phi=\int_{\Omega} u(-\Delta \phi)
$$

On the other hand, $v^{p} \in L^{\frac{p+1}{p}}(\Omega)$, and hence (see [13]) there exists a unique solution

$$
y \in W^{2, \frac{p+1}{p}}(\Omega) \quad \text { of } \quad-\Delta y=v^{p} .
$$

By the choice of $s$ we have $\frac{1}{p+1}>\frac{1}{2}-\frac{s}{N}$, which is equivalent to $\frac{1}{2}>\frac{p}{p+1}-\frac{s}{N}$, which in turn implies that $W^{2, \frac{p+1}{p}}(\Omega) \subset L^{2}(\Omega)$. Thus, we conclude that

$$
\int_{\Omega} v^{p} \phi=\int_{\Omega}(-\Delta y) \phi=\int_{\Omega} y(-\Delta \phi), \forall \phi \in H^{2}(\Omega) \cap H_{0}^{1}(\Omega)
$$

Comparing (20) and (21) yields

$$
\int_{\Omega}(y-u)(-\Delta \phi)=0, \forall \phi \in H^{2}(\Omega) \cap H_{0}^{1}(\Omega)
$$

and hence $u=y$; thus $u \in W^{2, \frac{p+1}{p}}(\Omega)$.

Consider now the second equation in system (7). Again, for $\psi \in H^{2}(\Omega) \cap H_{0}^{1}(\Omega)$ we have

$$
\int_{\Omega}(-\Delta \psi) v=\int_{\Omega} A^{t} \psi A^{s} v=\int_{\Omega} f(u) \psi, \forall \psi \in E^{t} .
$$

On the other hand, $E^{t} \subset\left\{u \in H^{t}(\Omega)|u|_{\partial \Omega}=0\right\} \subset C^{\lambda}(\Omega)$, with $\lambda=t-\frac{N}{2}$.

By our choices of $s$ and $t$ we have

$$
\begin{cases}1<t<2, & N=2 \\ \frac{3}{2}<t<2, & N=3\end{cases}
$$

and hence in both cases $u \in C^{\lambda}(\Omega)$ with $\lambda>0$. This implies that $f(u) \in L^{\infty}(\Omega)$, and hence there exists a unique solution

$$
w \in W^{2, q}(\Omega), \forall q \geq 1, \quad \text { of } \quad-\Delta w=f(u)
$$

Note that if $f \in C^{\lambda}$ and $\partial \Omega$ is sufficiently smooth, then $w \in C^{2, \lambda}(\Omega)$.

We finish by concluding as above that $w=v$, and that therefore $v \in W^{2, q}, \forall q \geq 1$, respectively $v \in C^{2, \lambda}(\Omega)$.

\section{Proof: the case $p \leq 1$}

In this section we consider the cases $0<p \leq 1(N=2,3)$ and $0<p<\frac{2}{N-2}(N \geq 4)$, i.e. we consider the situation where one equation has a sublinear nonlinearity in the form of a power, and the other equation has a superlinear nonlinearity.

\subsection{The functional}

We consider now the system

$$
\left\{\begin{array}{l}
-\Delta u=v^{p}, \quad \text { with } 0<p \leq 1 \\
-\Delta v=f(u)
\end{array}\right.
$$

System (22) can be written as

$$
\left\{\begin{array}{l}
(-\Delta u)^{1 / p}=v, \quad \text { with } 0<p \leq 1 \\
-\Delta v=f(u)
\end{array}\right.
$$


and thus we have the equivalent equation

$$
\left\{\begin{array}{l}
-\Delta(-\Delta u)^{1 / p}=-\Delta v=f(u) \\
u=\Delta u=0 \quad \partial \Omega
\end{array}\right.
$$

To equation (24) we may associate the following functional

$$
I(u)=\frac{p}{p+1} \int_{\Omega}|\Delta u|^{\frac{p+1}{p}}-\int_{\Omega} F(u) .
$$

Indeed, the derivative of $I(u)$ in direction $v$ yields

$$
I^{\prime}(u) v=\int_{\Omega}(-\Delta u)^{1 / p}(-\Delta v)-\int_{\Omega} f(u) v,
$$

and thus critical points of $I$ correspond to weak solutions of equation (23) and thus of system (22).

\subsection{Existence of critical points}

Note that the first term of the functional $I$ is defined on the space $E=W^{2, \frac{p+1}{p}}(\Omega) \cap W_{0}^{1, \frac{p+1}{p}}(\Omega)$. Since by assumption $p<\frac{2}{N-2}$ we have $\frac{p+1}{p}>1+\frac{N-2}{2}>\frac{N}{2}$, and thus

$$
W^{2, \frac{p+1}{p}}(\Omega) \subset \subset C(\Omega)
$$

Thus, the second term of the functional $I$ is defined if $F$ is continuous, and no growth restriction on $F$ is necessary. Since $F$ is differentiable, the functional $I$ is a well-defined $C^{1}$-functional on the space $E$.

We now show that the classical mountain-pass theorem of Ambrosetti-Rabinowitz may be applied to the functional $I$. Indeed, $I$ has a local minimum in the origin:

$$
I(u)=\frac{p}{p+1} \int_{\Omega}|\Delta u|^{\frac{p+1}{p}}-\int_{\Omega} F(u) \geq c \frac{p}{p+1}\|u\|_{C}^{\frac{p+1}{p}}-o\left(\|u\|_{C}^{\frac{p+1}{p}}\right)
$$

Next, let $u_{1}$ be any fixed element of $E$. Then

$$
I\left(s u_{1}\right) \leq \frac{p}{p+1} s^{\frac{p+1}{p}} \int_{\Omega}\left|\Delta u_{1}\right|^{\frac{p+1}{p}}-s^{\theta}\|u\|_{C}^{\theta}+d
$$

with $\theta>\frac{p+1}{p}$ (by assumption), and thus $I\left(s u_{1}\right) \rightarrow-\infty$ as $s \rightarrow \infty$.

Finally, we show that $I$ satisfies the Palais-Smale condition (PS). Let $\left(u_{n}\right) \subset E$ be a (PS)-sequence, i.e.

$$
\left|I\left(u_{n}\right)\right| \leq c \quad, \quad \text { and } \quad\left|I^{\prime}\left(u_{n}\right) v\right| \leq \epsilon_{n}\|v\|_{E}, \epsilon_{n} \rightarrow 0, \forall v \in E .
$$

We have

$$
\begin{aligned}
c+\epsilon_{n}\left\|u_{n}\right\|_{E} & \geq\left|\theta I\left(u_{n}\right)-I^{\prime}\left(u_{n}\right) u_{n}\right| \\
& \geq\left(\theta \frac{p}{p+1}-1\right) \int_{\Omega}\left|\Delta u_{n}\right|^{\frac{p+1}{p}}-\theta \int_{\Omega} F\left(u_{n}\right)+\int_{\Omega} f\left(u_{n}\right) u_{n} \\
& \geq\left(\theta \frac{p}{p+1}-1\right) \int_{\Omega}\left|\Delta u_{n}\right|^{\frac{p+1}{p}}-c \\
& \geq \delta\|u\|_{E}^{\frac{p+1}{p}}-c,
\end{aligned}
$$

and thus $\left(u_{n}\right)$ is bounded in $E$. Since $E$ is compactly imbedded in $C(\Omega)$, we find a convergent subsequence in $C(\Omega)$, and then it is standard to conclude that $u_{n}$ converges strongly also in E.

Thus, by the Mountain-Pass theorem we obtain a (non-trivial) critical point $u$, which gives rise to a solution to system (3). 


\subsection{Proof of Theorem 1.2}

The proof follows the same lines as in section 3.2. We just observe that for $\frac{2}{N-2} \leq p \leq 1$

$$
W^{2, \frac{p+1}{p}}(\Omega) \subset L^{\frac{N(p+1)}{N p-2(p+1)}}(\Omega) .
$$

The exponent $\frac{N(p+1)}{N p-2(p+1)}$ satisfies

$$
\frac{1}{p+1}+\frac{1}{\frac{N(p+1)}{N p-2(p+1)}}=1-\frac{2}{N},
$$

i.e. we are on the critical hyperbola. Hence, for $q+1<\frac{N(p+1)}{N p-2(p+1)}$ we are below the hyperbola, and we have $E \subset \subset L^{q+1}(\Omega)$ compactly. We can then proceed exactly as above, to obtain a critical point via the Mountain-Pass theorem.

\section{References}

[1] R. A. Adams, Sobolev Spaces, Academic Press (1975)

[2] A. Ambrosetti, P.H. Rabinowitz, Dual variational methods in critical point theory and applications, J. Functional Analysis 14 (1973), 349-381.

[3] Adimurthi, S.L. Yadava, Multiplicity results for semilinear elliptic equations in a bounded domain of $\mathbb{R}^{2}$ involving criitical exponent, Ann. Sc. Norm. Sup. Pisa XVII (1990), 481-504.

[4] H. Brezis, Analyse Fonctionelle, Masson, Paris, 1983.

[5] Ph. Clément, D.G. de Figueiredo, E. Mitidieri, Positive solutions of semilinear elliptic systems Comm. PDE 17(1992), 923-940.

[6] Ph. Clément, P. Felmer, E. Mitidieri, Homoclinic orbits for a class of infinite dimensional Hamiltonian systems, Ann. Scuola Norm. Sup. Pisa, Serie IV, Vol. XXIV, Fasc. 2 (1997), 367-393.

[7] D. G. de Figueiredo, J. M. do Ó and B. Ruf, On an inequality by N. Trudinger and J. Moser and related elliptic equations, Comm. Pure Appl. Math. 55 (2002), 135-152.

[8] D. G. de Figueiredo, J. M. do Ó and B. Ruf, Critical and subcritical elliptic systems in dimension two, Indiana University Mathematics Journal, to appear

[9] D. G. de Figueiredo and B. Ruf, On the existence and non-existence of solutions for elliptic equations with critical growth in $R^{2}$, Comm. Pure Appl. Math., 48, 1995

[10] D. G. de Figueiredo, O. H. Miyagaki and B. Ruf, Elliptic equations in $\mathbb{R}^{2}$ with nonlinearities in the critical growth range, Calc. Var. 3 (1995), 139-153.

[11] D. de Figueiredo and P. Felmer, On superquadratic elliptic systems. Trans. Amer. Math. Soc. 343 (1994), 99-116.

[12] P. Felmer, S. Martínez, Existence and uniqueness of positive solutions to certain differential systems, Adv. Diff. Equations, 4 (1998), 575-593.

[13] D. Gilbarg, N.S. Trudinger, Elliptic partial differential equations of second order, Springer, (1977)

[14] J. Hulshof, E. Mitidieri and R. vander Vorst, Strongly indefinite systems with critical Sobolev exponents, Trans. Amer. Math. Soc. 350 (1998), 2349-2365.

[15] J. Hulshof, and R. vander Vorst, Differential systems with strongly indefinite variational structure, J. Funct. Anal. 114 (1993), 32-58.

[16] J.L. Lions, E. Magenes, Non-homogeneous boundary value problems and applications: vol. I and II Springer-Verlag, Berlin, 1972

[17] S. Li, M. Willem, Applications of local linking to critical point thoery, J. Math anal. Appl. 189 (1995), 6-32.

[18] E. Mitidieri, A Rellich type identity and applications, Comm. Partial Diff. Equations 18, (1993), 125 - 151.

[19] J. Moser, A sharp form of an inequality by N. Trudinger, Ind. Univ. J. 20 (1971), 1077-1092. 
[20] S. I. Pohozaev, The Sobolev embedding in the case $p l=n$, Proceedings of the Technical Scientific Conference on Advances of Scientific Research 1964-1965. Mathematics Section, 158-170, Moscov. Ènerget. Inst., Moscow, 1965.

[21] P. H. Rabinowitz, Minimax Methods in Critical Point Theory with Applications to Differential Equations, CBMS Regional Conf. Ser. in Math., 65, AMS, Providence, RI, 1986.

[22] N. S. Trudinger, On imbeddings into Orlicz spaces and some applications, J. Math. Mech. 17 (1967), 473-484.

[23] R. vanderVorst, Variational identities and applications to differential systems, Arch. Rat. Mech. Anal. 116 (1991), 375-398.

Djairo G. de Figueiredo

IMECC

Universidade Estadual de Campinas

13081-970, Campinas, SP, Brazil

e-mail: djairo@ime.unicamp.br

Bernhard Ruf

Dipartimento di Matematica

Università degli Studi

Via Saldini 50, 20133 Milano, Italy

e-mail: ruf@mat . unimi.it 